\title{
BEN/SC1/DM-GRASP Expression during Neuromuscular Development: a Cell Adhesion Molecule Regulated by Innervation
}

\author{
C. Fournier-Thibault, ${ }^{1,2}$ O. Pourquié, ${ }^{3}$ T. Rouaud, ${ }^{1}$ and N. M. Le Douarin ${ }^{2}$ \\ ${ }^{1}$ Centre National de la Recherche Scientifique (CNRS) EP 1593, Faculté des Sciences et des Techniques, BP 92208, \\ 44322 Nantes Cedex 03, France, 2Institut d'Embryologie cellulaire et Moléculaire du CNRS et du Collège de France, \\ 94736, Nogent sur Marne Cedex, France, and 3/nstitut de Biologie du Développement de Marseille, Laboratoire de \\ Génétique et Physiologie du Développement-Unité Mixte de Recherche CNRS 6545, Campus de Lumigny, Case 907, \\ 13288, Marseille Cedex 09
}

\begin{abstract}
BEN/SC1/DM-GRASP is a cell adhesion molecule belonging to the Ig superfamily that is transiently expressed during avian embryogenesis in a variety of cell types, including the motoneurons of the spinal cord. We have investigated the pattern of BEN expression during neuromuscular development of the chick. We show that both motoneurons and their target myoblasts express BEN during early embryonic development and that the protein becomes restricted at neuromuscular contacts as soon as postsynaptic acetylcholine receptor clusters are observed in muscle fibers. Muscle cells grown in vitro express and maintain BEN expression even when they fuse and give rise to mature myotubes. When embryos are deprived of innervation by neural tube ablation, BEN expression is observed in muscle fibers, whereas, in control, the protein is already restricted at neuromuscular synaptic sites. These results demonstrate that all myogenic
\end{abstract}

cells intrinsically express BEN and maintain the protein in the absence of innervation.

Conversely, when neurons are added to myogenic cultures, BEN is rapidly downregulated in muscle cells, demonstrating that innervation controls the restricted pattern of BEN expression seen in innervated muscles. After nerve section in postnatal muscles, BEN protein becomes again widely spread over muscle fibers. When denervated muscles are allowed to be reinnervated, the protein is reexpressed in regenerating motor axons, and reinnervation of synaptic sites leads to the concentration of BEN at neuromuscular junctions.

Our results suggest that BEN cell adhesion molecule acts both in the formation of neuromuscular contacts during development and in the events leading to muscle reinnervation.

Key words: adhesion molecule; neuromuscular development; synaptic sites; nerve control; chick embryo; cell recognition
During embryonic development, innervation of skeletal muscles takes place according to a precise pattern. In the chick embryo, motor axons are segmentally segregated into spinal nerves by restricted growth through the anterior half of the sclerotomes (Keynes and Stern, 1984, 1988; Tosney, 1987, 1988). In the forelimb, the main nerve trunks enter the dorsal and ventral muscle masses at embryonic day 4 (E4; Noakes et al., 1986), and, at E6, the first neuromuscular synapses can be detected by the clustering of the acetylcholine receptors (AChRs) at endplates (Smith and Slater, 1983; Fallon and Gelfman, 1989). Specialization of the neuromuscular junction progresses throughout development, including the assembly of the synaptic basal lamina and the reduction of extrasynaptic AChR density (for review, see Hall and Sanes, 1993).

Evidence has been accumulated showing that guidance cues are critical for the establishment of nerve-muscle connections.

\footnotetext{
Received June 8, 1998; revised Dec. 3, 1998; accepted Dec. 7, 1998.

This work was supported by the Association Française contre les Myopathies and the Centre National de la Recherche Scientifique. We thank C. Corbel and A. Burns for critical reading of this manuscript. We thank E. Pollerberg for the generous gift of the 4H5 antibody. We are grateful to Françoise Viala, Géraldine Boursier, and Sophie Gournet for the illustrations.

Correspondence should be addressed to C. Fournier-Thibault, Centre National de la Recherche Scientifique Unité Mixte de Recherche 7622, Université Pierre et Marie Curie, Batiment. C, 7 ème étage, 9 quai St. Bernard, 75252, Paris Cedex 05, France.

Dr. Fournier-Thibault's present address: Centre National de la Recherche Scientifique Unité Mixte de Recherche 7622, Université Pierre et Marie Curie, 9 quai St. Bernard, 75252, Paris Cedex 05, France.

Copyright (ㄷ) 1999 Society for Neuroscience $\quad 0270-6474 / 99 / 191382-11 \$ 05.00 / 0$
}

Contribution of cell adhesion molecules (CAMs) has been demonstrated at different steps of the innervation process (Chiba and Keshishian, 1996; Fields and Itoh, 1996; Schuster et al., 1996). In the chick, the segmental motor axon projections across the sclerotome were shown to be regulated by T-cadherin and tenascin (Martini and Schachner, 1991; Fredette and Ranscht, 1994). The polysialilated (PSA) form of $N$-CAM is highly expressed on axons when they are branching in the muscle (Landmesser et al., 1990), and enzymatic removal of PSA results in increased fasciculation and reduced nerve branching (Landmesser et al., 1990; Tang et al., 1994). Neuromuscular blockade induces an increase in neuronal PSA NCAM expression, suggesting that downregulation of this molecule is necessary for stabilization of neuromuscular junctions (Landmesser et al., 1990; Bruses et al., 1995).

$\mathrm{BEN} / \mathrm{SC} 1 / \mathrm{DM}-\mathrm{GR}$ ASP is a member of the CAM Ig superfamily that has been discovered independently by different groups (SC1: Tanaka and Obata, 1984; Tanaka et al., 1991; BEN: Pourquié et al., 1990, 1992a; DM-GRASP: Burns et al., 1991). This molecule is characterized by an extracellular domain carrying two V-type followed by three C2-type Ig-like motifs. BEN is transiently expressed during avian embryogenesis, particularly by cells of the nervous and hemopoietic systems and certain epithelia (Pourquié et al., 1990, 1992a,b; Corbel et al., 1992a,b, 1996). During the development of the nervous system, the protein is transiently observed on neurons of the peripheral nervous system, motoneurons of the spinal cord and the brain (Pourquié et al., 1990, 1992a; Simon et al., 1994; Chedotal et al., 1996), cerebellar 
climbing fibers (Pourquié et al., 1992b; Chedotal et al., 1996), and retinotectal projections (Pollerberg and Mack, 1994).

Monoclonal antibodies against BEN perturb axonal growth of sympathetic neurons while the purified protein selectively supports neurite extension in vitro from a subset of neuronal cell types (Burns et al., 1991; DeBernardo and Chang, 1995, 1996). BEN has been shown to have homophilic properties as demonstrated by cell adhesion experiments on substrates and in vitro assays (Tanaka et al., 1991; El-Deeb et al., 1992; DeBernardo and Chang, 1995; Corbel et al., 1996). However, heterophilic interactions have also been described, notably with $\mathrm{Ng}$-CAM during extension of neurites from sympathetic neurons (DeBernardo and Chang, 1996) and with CD6 in the hemopoietic system (Bowen et al., 1995, 1997).

The dynamic expression of BEN during development of motor axons (Pourquié et al., 1990, 1992a) led us to investigate its pattern of expression during neuromuscular development of the chick. We show here that both motoneurons and their target, the myoblasts, express BEN during early embryonic development and that the protein becomes later on restricted at the neuromuscular contacts as soon as postsynaptic AChR clusters are observed. Furthermore, we demonstrate that BEN expression in muscles is regulated by the contact with nerve endings during embryonic as well as postnatal development, suggesting that this molecule contributes both to the formation of neuromuscular contacts and to the events leading to muscle reinnervation.

\section{MATERIALS AND METHODS}

Experiments were performed using JA57 chick embryos or chickens. Embryos were staged according to Hamburger and Hamilton (1951).

Cell culture. Primary muscle cell cultures were prepared from ventral and dorsal muscle masses of the forelimb removed from E6 chick embryos. Cells were seeded after mechanical dissociation at a density of $10^{5}$ myoblasts per milliliter of medium. Cultures were routinely grown in DMEM supplemented with $10 \%$ fetal calf serum and $1 \%$ chick serum from $24 \mathrm{hr}$ to $6 \mathrm{~d}$.

For cocultures of neurons and muscle cells, the ventral part of the neural tube was excised from E2 chick embryos using Pancreatin (Life Technologies, Grand Island, NY) transferred to DMEM supplemented with $10 \%$ fetal calf serum and $1 \%$ chick serum and then mechanically dissociated. Cells of the neural tube were added to myoblasts grown in vitro for $2 \mathrm{~d}$ at a density of $5.10^{3}$ neural cells per milliliter of medium and cocultivated for $48 \mathrm{hr}$ to $6 \mathrm{~d}$.

Neural tube ablation. Fertilized chick eggs were incubated at $37^{\circ} \mathrm{C}$ for $2 \mathrm{~d}$ to stage 14 of Hamburger and Hamilton (1951), corresponding to 20 somites. The portion of the neural tube comprising the region between somites 15 and 20 , which gives rise to the brachial innervation, was removed microsurgically after a brief Pancreatin treatment. Chick embryos were then reincubated at $37^{\circ} \mathrm{C}$ and allowed to develop until E8 or E10.

Denervation procedure. Unilateral (left) denervation of latissimus dorsi muscles of the wing [anterior latissimus dorsi (ALD) and posterior latissimus dorsi (PLD)] was performed aseptically in 4-d-old chickens under anesthesia. A 5-8 mm segment was removed from the common nerve trunk according to the method of Khaskiye et al. (1986). In some cases, muscles were removed $10 \mathrm{~d}$ after denervation, and the lack of reinnervation was ascertained by staining muscle sections with a monoclonal antibody directed against the $68 \mathrm{kDa}$ neurofilament subunit (NF; Sigma, St. Louis, MO), as described in the immunocytochemical section. In other cases, muscles were removed 15-20 d after denervation to allow their reinnervation to occur. Reinnervation was also checked by using the anti-neurofilament antibody.

In situ hybridization. A $1.5 \mathrm{~kb}$ SacII-HapII fragment encompassing the Ig-like domains of the BEN molecule was cloned in a pGEM-3Z vector (Promega, Madison, WI) and used to prepare antisense and sense BEN-specific probes.

For radioactive in situ hybridization, embryos were fixed at various stages between E3 and E15 in Carnoy's solution, embedded in paraffin, and transversally sectioned $(5-\mu \mathrm{m}$-thick). Section treatment, hybridiza- tion, and washing were performed according to the procedure described by Eichmann et al. (1993). Sense and antisense probes were hybridized at $5.10^{4} \mathrm{cpm} / \mu \mathrm{l}$ of hybridization buffer. After hybridization, washed sections were dehydrated and autoradiographed with NBT-2 emulsion (Eastman Kodak, Rochester, NY) and then exposed for 8-15 d.

For nonradioactive hybridization, sense and antisense probes were synthesized in the presence of digoxygenein-UTP (Boehringer Mannheim, Indianapolis, IN) using a Promega transcription kit. For wholemount in situ hybridization, embryos were fixed in $4 \%$ formaldehyde with $2 \mathrm{mM}$ EGTA, progressively dehydrated in methanol, and stored at $-20^{\circ} \mathrm{C}$. Hybridization was performed on rehydrated embryos at $70^{\circ} \mathrm{C}$ according to the procedure of Henrique et al. (1995). After posthybridization washes, embryos were incubated overnight in alkalinephosphatase-coupled anti-digoxygenin antibody (Boehringer Mannheim) and stained for $12-24 \mathrm{hr}$.

For nonradioactive in situ hybridization on cryostat sections, embryos (between E3 and E15) or postnatal muscles were fixed in $4 \%$ paraformaldehyde, $4 \%$ sucrose in $0.12 \mathrm{M}$ phosphate buffer, embedded in a $7.5 \%$ gelatin-15\% sucrose solution and frozen in liquid nitrogen-cooled isopentane. Transverse serial sections for embryos or longitudinal sections for muscles (15- $\mu \mathrm{m}$-thick) were collected on Superfrost/Plus slides (Fisher Scientific, Houston, TX), and hybridization was performed according to the procedure of Strähle et al. (1994). For hybridization on cultures, cells were rinsed in PBS, fixed in $4 \%$ paraformaldehyde, and hybridized using the same procedure as for embryo or muscle sections.

Immunocytochemistry. For immunocytochemistry, cryostat sections were prepared from E3 to E15 embryos or posthatched muscles as described above for in situ hybridization.

BEN protein detection was performed using a mouse monoclonal BEN antibody (Pourquié et al., 1990; diluted 1:10,000) or a rabbit polyclonal BEN antibody (4H5, diluted 1:500, generous gift from Dr. E. Pollerberg; Pollerberg and Mack, 1994), which was revealed with fluoresceinconjugated or biotinylated anti-mouse or anti-rabbit secondary antibodies (1:100; Southern Biotechnology, Alabaster, AL). When biotinylated secondary antibodies were used, sections were processed with the ABC solution (1:100; Vector Laboratories, Burlingame, CA), and peroxidase was revealed using diaminobenzidine as a chromogen.

Muscle cells in vitro were visualized by their myosin content. Myosin detection on cultures was performed using a monoclonal anti-fast myosin (Sigma; diluted 1:100) or a monoclonal antibody against the sarcomeric myosin (MF20; Developmental Hybridoma Bank, University of Iowa, Iowa City, IA). Cultures were rinsed in PBS, fixed in $4 \%$ paraformaldehyde, and simultaneously treated with $4 \mathrm{H} 5$ polyclonal and MF20 or fast-myosin monoclonal antibodies that were revealed with a fluoresceinconjugated anti-rabbit and a rhodamine isothiocyanate-labeled antimouse secondary antibody (diluted 1:100; Southern Biotechnology).

AChR clusters were detected by incubation in tetramethylrhodamineconjugated $\alpha$-bungarotoxin (TRITC- $\alpha$ BGT; Molecular Probes, Eugene, OR), and sections or cultures were simultaneously incubated with NF, $\mathrm{BEN}$, or $4 \mathrm{H} 5$ antibodies to visualize nerve terminals.

Immunoblotting. Proteins were extracted from contralateral and denervated muscles of 14-d-old denervated chickens. Samples, which required $\sim 20$ muscles, were collected from both ALD and PLD. Muscles were crushed in $1 \%$ Triton X-100 PBS buffer containing protease inhibitors (Sigma, diluted 1:100). After centrifugation at 20,000 $\times g$ at $4^{\circ} \mathrm{C}$ for 20 min, the supernatant was diluted twice in $10 \%$ SDS, $10 \%$ glycerol sample buffer. Immunoblotting was performed as previously described (Lefeuvre et al., 1996) under nonreducing conditions using the different antiBEN antibodies diluted 1:100. Biotin-streptavidin reagent was used for detection of anti-mouse IgG1 or anti-rabbit Ig biotin. After peroxidase revelation, the antigen-antibody complexes were visualized using enhanced chemoluminescence (ECL) detection (Amersham kit). Tests for immunochemical specificity were performed by omission of primary $\mathrm{mAbs}$ and use of preabsorbed controls.

\section{RESULTS}

\section{BEN expression during in vivo neuromuscular development of the chick}

The expression of BEN mRNA and protein was studied at the brachial level from the time of dermamyotome formation in the embryo until 4 weeks after hatching when mature neuromuscular junctions are definitively established. 
BEN mRNA appears in brachial chick somites at stage 15 of Hamburger and Hamilton (1951) (25 somites, Fig. 1A). The protein can be identified by $4 \mathrm{H} 5$ antibody, which stains the dermamyotomal compartment of the somite (Fig. $1 B$ ). BEN is also detected in cell bodies of motoneurons at this stage (Fig. $1 A, B$; Pourquié et al., 1990). At E5, BEN mRNA and protein appear in dorsal and ventral muscle masses of the forelimb bud (Fig. 1C,D). Simultaneously, the main nerve trunks coming from the ventral spinal roots begin to enter the limb buds and react with $4 \mathrm{H} 5$ antibody (Fig. 1D) while the protein progressively decreases on the soma of motoneurons (data not shown, see Pourquié et al., 1990). At E7, brachial muscles of the forelimb are individualized and express BEN, however, both mRNA and protein are restricted to a subset of fibers within each muscle (Fig. $1 E, G)$. BEN protein is observed on intramuscular nerve terminals (Fig. $1 F, G$ ), and the first signs of colocalization with AChR clusters are detected (Fig. $1 F-H)$. In these nerve-contacted myotubes, BEN protein has disappeared from the muscle fiber and is restricted to synaptic sites while it is still expressed in noninnervated muscle cells (Fig. $1 F-H)$. This dynamic expression of BEN protein could be related to the downregulation of the mRNA observed in some muscle fibers at this stage (Fig. $1 E$ ). At the end of the embryonic period, myotubes are nearly devoid of BEN mRNA (Fig. 1I), which is still present on motoneuron cell bodies (data not shown), and a perfect overlap between AChR clusters and BEN protein is observed at all synaptic sites $($ Fig. $1 J, K)$. Such a colocalization is maintained until the adult stage.

Thus, at early stages of development, both motoneurons and their target muscle cells express BEN mRNA and protein. During further embryonic development, when BEN protein is progressively restricted to growth cones of motor axons, it disappears from the surface of myotubes to be finally exclusively located at the neuromuscular junctions.

\section{BEN expression during in vitro myogenesis}

Myogenic cultures were prepared from dorsal and ventral muscle primordia of E6 forelimb buds. At this stage, these muscle primordia are not yet innervated. Twenty four hours after plating, BEN mRNA and protein are detected in myoblasts (Fig. 2A,C). Double labeling of cultures with fast myosin and $4 \mathrm{H} 5$ antibodies shows that postmitotic myoblasts express BEN (Fig. 2C,D). After $6 \mathrm{~d}$ of culture, myoblasts have fused, and numerous myotubes are differentiated that strongly accumulate BEN mRNA and protein (Fig. $2 B, E$ ). Muscles cells in vitro are therefore capable of expressing BEN and maintaining this expression in the absence of innervation.

\section{Role of motor innervation on BEN expression during muscle development}

The influence of innervation on muscular expression of BEN was studied by different approaches. We looked at the effect of neurons on BEN accumulation in muscle cells grown in vitro. The role of innervation was also tested during development in vivo, first, by analyzing the effects of neural tube removal on BEN expression during myogenesis, and second, by looking at the influence of motor nerve section and subsequent muscle reinnervation on BEN expression in postnatal muscles.

\section{$B E N$ expression in myoblasts cocultivated in vitro with neurons}

Neurons prepared from the ventral part of E2 chick embryo neural tubes were added to brachial myoblasts previously cultured for $2 \mathrm{~d}$, at a time when they strongly express BEN. Forty eight hours later, axons originating from isolated nerve cells or neuron clusters have sprouted and invaded myogenic cultures (Fig. $2 F, G)$. At this time, BEN mRNA is accumulated in neuronal cell bodies (Fig. $2 F$ ) and BEN protein present at the surface of axons (Fig. 2G). Myotubes, which begin to form, are contacted by nerve terminals, and the accumulation of both mRNA and BEN protein is largely reduced in these innervated muscle cells (Fig. $2 F, G$ ) when compared with aneural cultures (Fig. $2 A-E$ ). Simultaneous treatment with TRITC- $\alpha$ BGT and $4 \mathrm{H} 5$ antibody shows that clustering of AChR has not yet occurred at this time (Fig. 2G,H). From $6 \mathrm{~d}$ of coculture of neurons and muscle cells, colocalization of BEN-positive nerve profiles with TRITC- $\alpha$ BGT sites is observed on contacted muscle fibers in which BEN protein has been downregulated (Fig. 2I) when compared with myotubes differentiated in vitro without neurons (Fig. $2 E$ ).

Our results show that when neurons are added to muscle cells grown in vitro, which normally maintain BEN expression during their maturation, innervation induces a downregulation of both mRNA and protein in myotubes, as observed during normal embryonic development.

\section{BEN expression in embryonic muscles developing in aneural conditions}

Neural tube ablation was performed to assess its possible influence on BEN muscular expression in ovo. Ablation was performed at the 20 somite stage at the level of the last five somites to suppress the neural tube portion innervating the brachial muscles. Efficiency of the excision was assessed by treating serial sections from experimental embryos with anti-neurofilament or BEN antibodies. Twenty embryos (10 at E6 and 10 at E8) in which neural tubes had been successfully removed were analyzed. At E8, the region located between the 13th and the 19th vertebrae was dissected, transversally cut along its whole length, and the appearance of the first cells in the nodose ganglia was systematically taken as an anatomical reference in control and experimental embryos. As previously described, when the neural tube is removed and the notochord left in place, myotomes develop and give rise to paraxial muscles that degenerate during the second week of embryogenesis because of the lack of innervation (Rong et al., 1992; Lefeuvre et al., 1996). At E8, BEN mRNA in control embryos is downregulated in most axial muscle fibers (Fig. 3A). This may be related to the fact that at this age, most epaxial muscles are already innervated. Indeed, the timing of innervation of myotome-derived muscles in the chick precedes by $\sim 2 \mathrm{~d}$ the establishment of neuromuscular contacts in limb muscles (AudaBoucher et al., 1997). In contrast, at E8 in experimental embryos, BEN mRNA is still abundant in all fibers of epaxial muscles (Fig. $3 B)$. In the same way, aneural muscles in the forelimb exhibit an homogenous expression of BEN mRNA (Fig. 3C), while in controls, some already innervated myotubes of the hypaxial muscles have downregulated their BEN mRNA level at this time (Fig. $1 E)$. The persistence of BEN mRNA in aneural muscles is accompanied by an accumulation of the protein at the membrane of muscle cells (data not shown).

Thus, BEN expression in muscles of embryos deprived of neural tube fails to be downregulated during development in ovo in the absence of innervation. Taken together, these and the in vitro results indicate that during embryogenesis, the expression of $\mathrm{BEN}$ in muscle cells is nerve-dependent, being largely repressed when BEN-positive axon terminals progressively contact embryonic muscle fibers. 

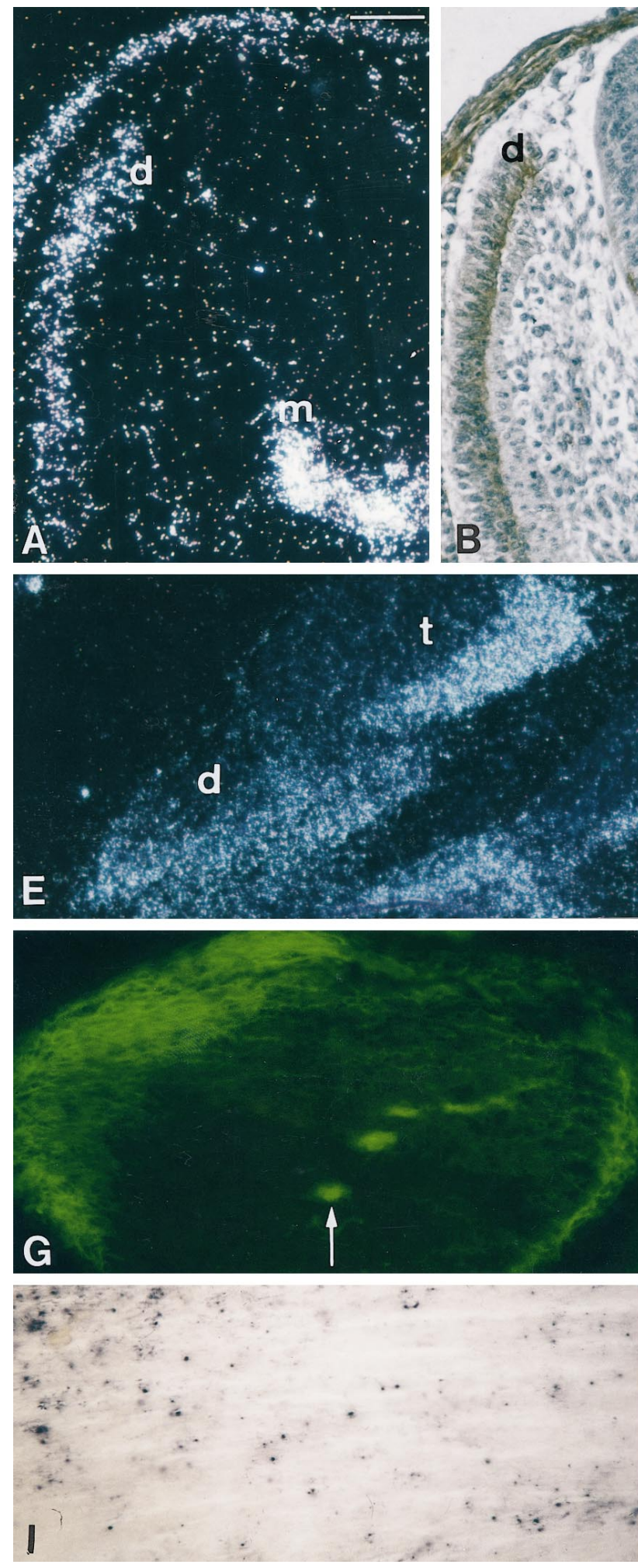
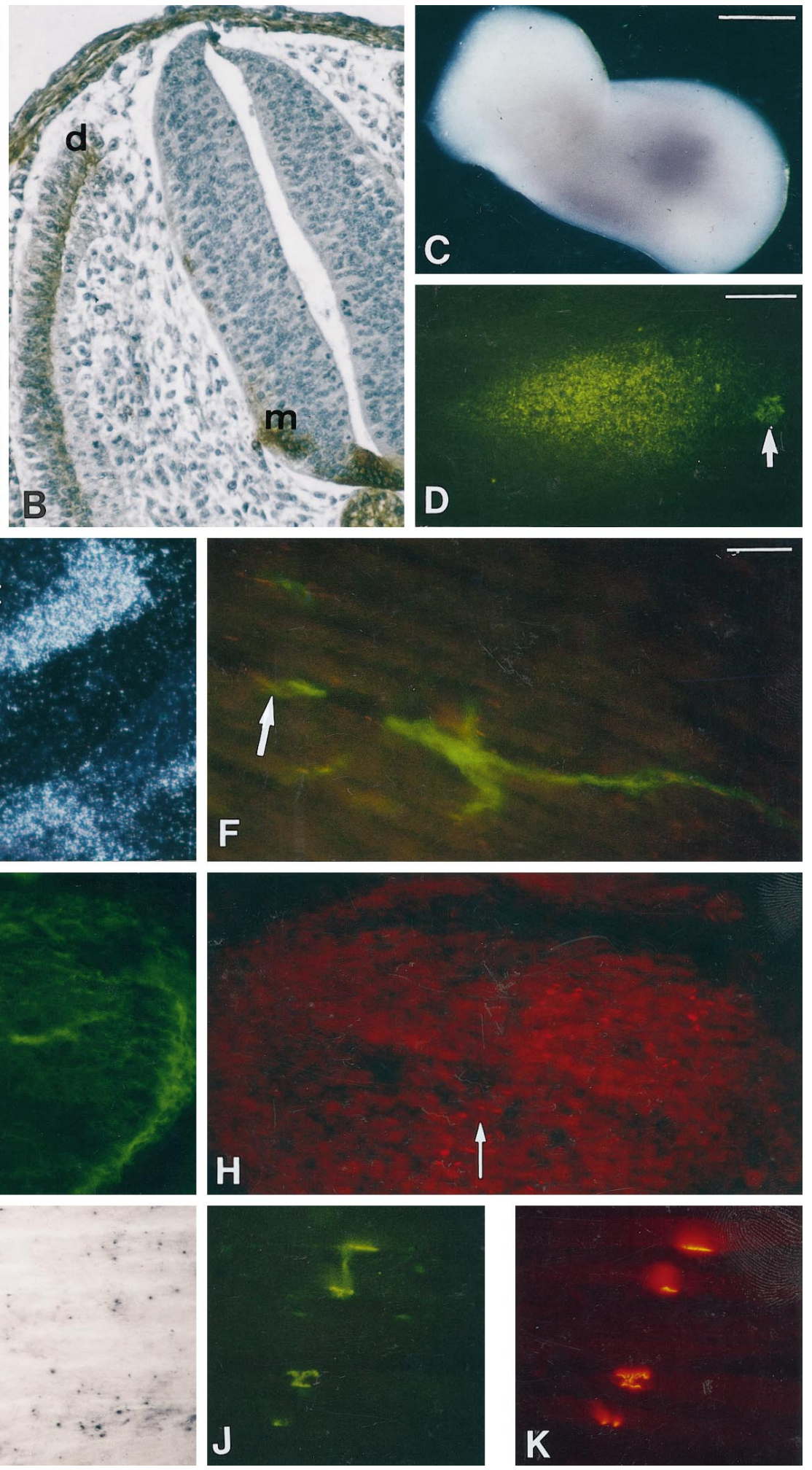

Figure 1. BEN expression during neuromuscular development in chick embryo. $A, B, 25$ somite stage embryo: labeling of serial transverse sections at the brachial level with BEN probe $(A)$ and $4 \mathrm{H} 5$ antibody $(B)$. BEN is detected in motoneurons $(m)$ and dermamyotome $(d)$. $C, D$, E5 stage: whole-mount in situ hybridization of the forelimb bud with BEN probe $(C)$ and $4 \mathrm{H} 5$ antibody staining on a transverse section at the level of the ventral muscle primordia $(D)$. Dorsal and ventral muscle masses express BEN mRNA and protein; motor nerve trunks that begin to invade the bud are also stained with BEN antibody $(D$, arrow). $E-H$, E7 stage: in situ hybridization with BEN probe on a transverse section at the wing level ( $E$; $t$, triceps muscle and $d$, deltoid muscle) Simultaneous staining with $4 \mathrm{H} 5$ antibody $(F, G)$ and TRITC- $\alpha$ BGT $(F, H)$ on the same transverse $(G, H)$ or longitudinal $(F)$ sections from triceps muscle. BEN mRNA and protein appear restricted to a subset of fibers within a muscle. Intramuscular nerve terminals express BEN protein, and the first signs of colocalization with AChR clusters are observed $(F-H$, arrows). $I-K$, E18 stage: in situ hybridization with BEN probe $(I)$ and simultaneous detection of $4 \mathrm{H} 5$ antibody $(J)$ and TRITC- $\alpha$ BGT $(K)$ on the same longitudinal section from PLD wing muscle. Myotubes appear nearly devoid of BEN messenger, whereas BEN protein and AChR clusters perfectly overlap at neuromuscular junctions. Scale bars: $C, 400 \mu \mathrm{m} ; A, B, E, 100 \mu \mathrm{m} ; D, G-K, 60 \mu \mathrm{m} ; F, 40 \mu \mathrm{m}$. 

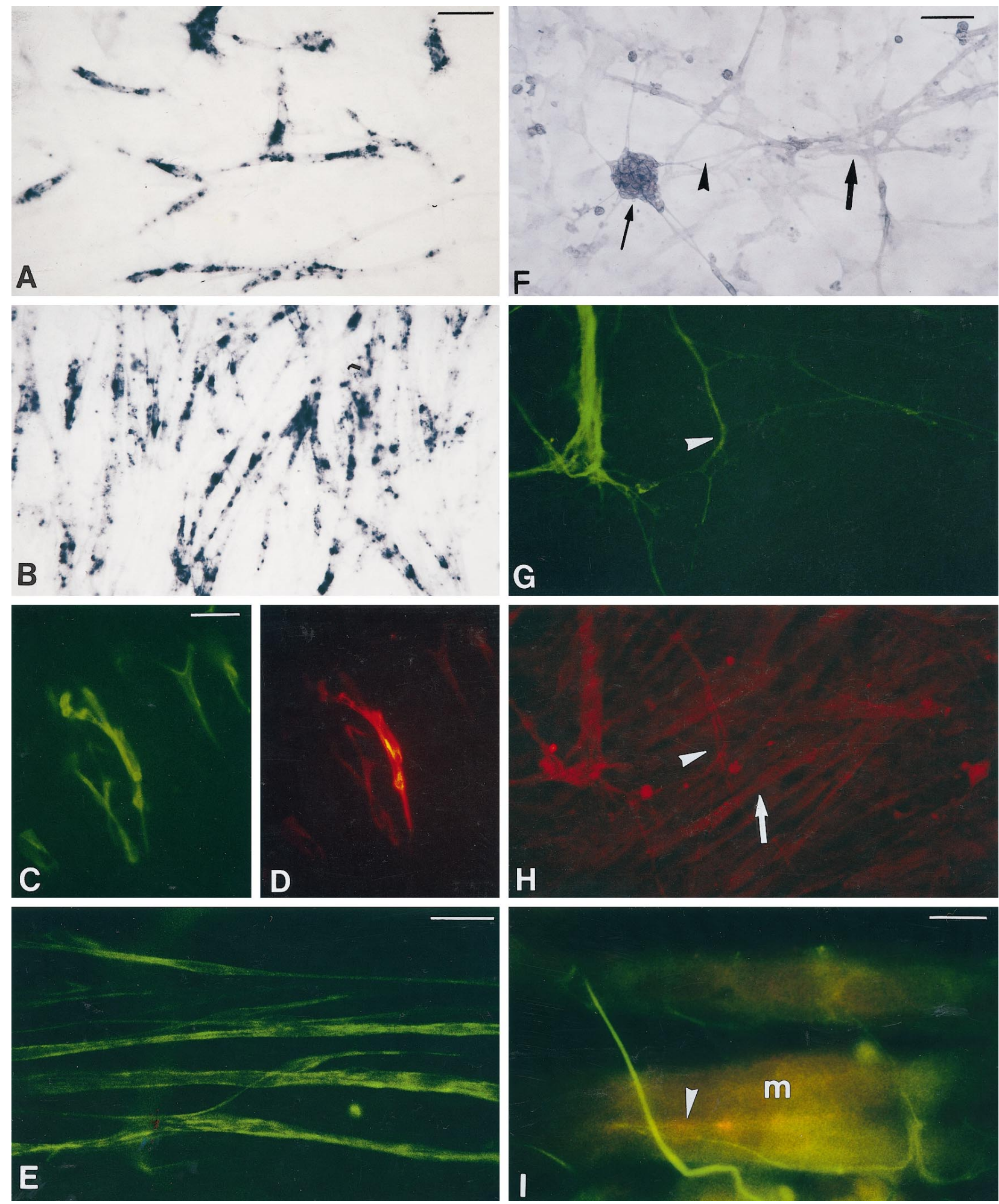

Figure 2. BEN expression during in vitro myogenesis. $A-E$, In vitro myogenesis without neurons. $A, C, D$, Muscle cells cultivated for 24 hr: in situ hybridization with BEN probe $(A)$, simultaneous labeling with 4H5 $(C)$, and fast-myosin antibodies $(D)$ in the same culture. Proliferating and postmitotic myoblasts express both BEN mRNA and protein. $B, E$, Muscle cells cultivated for 6 d: in situ hybridization with BEN probe $(B)$; $4 \mathrm{H} 5$ antibody staining $(E)$. Numerous myotubes are differentiated, expressing both BEN mRNA and protein. $F-I$, In vitro myogenesis with neurons. $F-H$, Myoblasts and neurons cocultivated for $3 \mathrm{~d}$ : in situ hybridization with BEN probe $(F)$ and simultaneous labeling with $4 \mathrm{H} 5$ antibody $(G)$ and TRITC- $\alpha$ BGT $(H)$ in the same culture. BEN mRNA is accumulated in cell bodies of motoneurons ( $F$, thin arrow), whose axons $(F-H$, arrowheads) have invaded myogenic cultures and express BEN protein $(G)$. Conversely, both mRNA $(F)$ and protein $(G)$ are downregulated in muscle fibers $(F, H$, large arrows) in which clustering of AChR has not yet occurred at this time $(H)$. I, Simultaneous staining with $4 \mathrm{H} 5$ antibody and TRITC- $\alpha$ BGT in myotubes and neurons cocultivated for $6 \mathrm{~d}$. BEN-positive axons begin contacting AChR clusters (arrowhead; $m$, myotube). Scale bars: $F-H, 125 \mu \mathrm{m} ; A, B, 60 \mu \mathrm{m} ; E, 45 \mu \mathrm{m} ; C, D, 30 \mu \mathrm{m}$; I, $10 \mu \mathrm{m}$. 


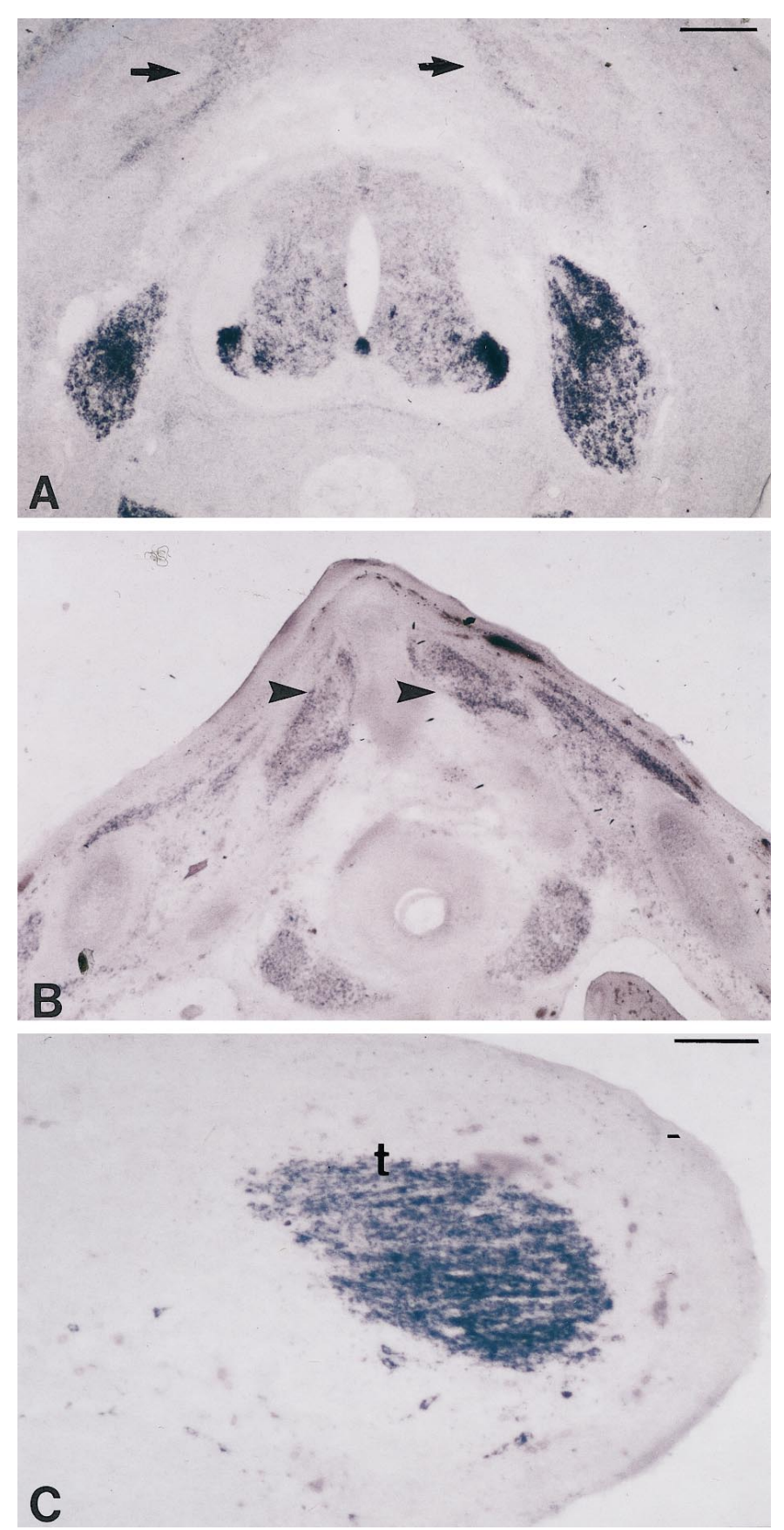

Figure 3. BEN expression in embryonic muscles after neural tube ablation. In situ hybridization with BEN probe on transverse sections from the brachial level of E8 control $(A)$ and neural tube-ablated $(B, C)$ embryos. At this stage, BEN mRNA is nearly repressed in most fibers of axial muscles in control ( $A$, arrows), whereas it is expressed in all fibers of axial muscles from the same level in neural tube-ablated embryos of the same age $(B$, arrowheads). Aneural muscles in the forelimb also exhibit an homogeneous expression of BEN mRNA in all muscle cells ( $C$; $t$, triceps). Scale bars: $A, B, 200 \mu \mathrm{m} ; C, 80 \mu \mathrm{m}$.

\section{Influence of innervation on the synaptic expression of BEN during postnatal neuromuscular development}

The role of motor nerve on BEN expression in mature muscle fibers was tested by denervating brachial muscles in young chicks. Two muscles of the wing were chosen, the ALD and PLD, because of their particular pattern of innervation. In the adult, the PLD is characterized by a unique synaptic site per muscle fiber, whereas the mature ALD maintains a multiplicity of neuromuscular contacts on each myotube (Ginsborg and Mackay, 1960). These muscles therefore represent a convenient model to study the regulation of BEN expression at neuromuscular junctions.

The common nerve trunk of the latissimus dorsi muscles was severed in 4-d-old chickens, and denervated muscles were removed $10 \mathrm{~d}$ later. Contralateral muscles were taken as controls. Sections were performed at different levels for each denervated muscle and incubated with an anti-NF antibody to check denervation efficiency. Serial sections were treated for AChR clusters and BEN expression analysis. Thirty well denervated muscles (15 ALD and 15 PLD) were used for the study. When the common latissimus dorsi nerve trunk was severed in posthatched chicks, BEN mRNA accumulation was observed in denervated muscles $10 \mathrm{~d}$ after the operation (Fig. 4A), whereas it had been largely downregulated in control muscles at this age. Accumulation of BEN mRNA leads to a reappearance of the protein all along the membrane of denervated muscle fibers (Fig. $4 B$ ). BEN mRNA and protein are observed in both denervated ALD and PLD muscles but to a lesser extent in multiinnervated ALD. Simultaneous revelation of $4 \mathrm{H} 5$ antibody and TRITC- $\alpha$ BGT shows that the wide distribution of BEN protein at the surface of denervated myotubes is synchronous with the characteristic dispersal of AChR clusters observed in both muscles after nerve section (Fig. $4 C$ ).

When operated chickens are allowed to develop for 2 weeks after denervation, a regeneration of the sectioned motor nerve occurs, inducing a progressive reinnervation of denervated muscles. Reinnervation was verified by the anti-NF-positive reaction of sections from different levels of experimental muscles. Hybridization of adjacent sections with BEN probe reveals that mRNA is downregulated in reinnervated muscles when compared with denervated ones (Fig. 4D). Staining with $4 \mathrm{H} 5$ antibody shows that regenerating nerve terminals express BEN protein (Fig. $4 E$ ). Simultaneously, the accumulation of the protein at the muscle membrane is progressively downregulated (Fig. $4 E$ ). Treatment of the same sections with TRITC- $\alpha$ BGT reveals that most AChRs are still dispersed on muscle membrane at this stage (Fig. $4 F$ ), showing that the clustering of receptors in reinnervated muscle fibers occurs after BEN protein downregulation. Colocalization of TRITC- $\alpha$ BGT sites and BEN-positive nerve terminals only begins from 3 weeks after the operation (Fig. $4 G$ ).

Thus, the dynamic expression of BEN in denervated postnatal muscles is similar to that observed in muscle cells of neural tube-ablated embryos and of normal embryos before myofibers are contacted by nerve terminals. In addition, during muscle reinnervation, the pattern of BEN expression recapitulates the events observed during embryonic development. The regenerating motor axons express BEN, and a downregulation of the protein is observed on reinnervated muscle fibers. Taken together, these observations underline the importance of innervation in the regulation of BEN expression in muscle fibers and suggests a role for this molecule both during the formation of neuromuscular contacts and in the events of muscle reinnervation.

\section{Characterization of BEN protein in muscle fibers}

To characterize BEN protein accumulation in muscle cells and at neuromuscular junctions, Western blots were performed with extracts from control and denervated ALD and PLD muscles. Latissimus dorsi muscles of 4-d-old chickens were unilaterally 

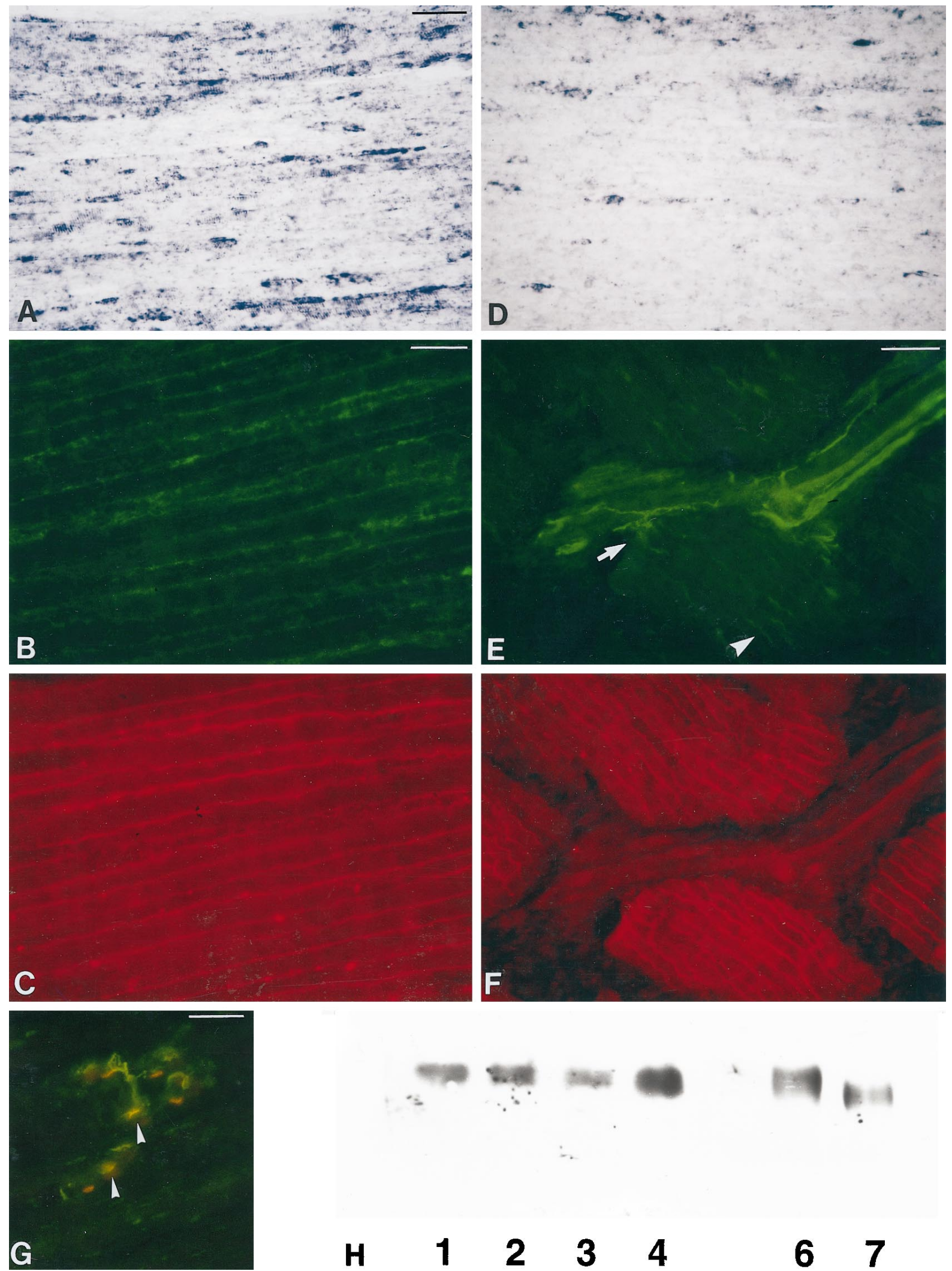

67

Figure 4. Effects of muscle denervation and reinnervation on BEN expression during neuromuscular postnatal development. $A-C$, Longitudinal sections of PLD muscle from a 14-d-old chicken in which the common LD nerve has been severed $10 \mathrm{~d}$ before: in situ hybridization with BEN probe $(A)$ and simultaneous staining of a serial section with $4 \mathrm{H} 5$ antibody $(B)$ and TRITC- $\alpha$ BGT $(C)$. Denervation induces an accumulation of BEN mRNA and protein at the surface of muscle fibers, which is synchronous of AChR cluster dispersal. $D-G$, Longitudinal sections of PLD muscle from a 20-d-old chicken in which the common LD nerve has been severed $16 \mathrm{~d}$ before and allowed to regenerate and reinnervate the muscle: in situ hybridization with BEN probe $(D)$ and simultaneous staining of a serial section with $4 \mathrm{H} 5$ antibody $(E)$ and TRITC- $\alpha$ BGT $(F)$. Progressive reinnervation of denervated muscle leads to a downregulation of BEN mRNA and a decrease of the protein at the membrane of muscle fibers (Figure legend continues.) 
denervated and removed 10 d later. One cryostat-cut section was performed at different levels of denervated muscles to ascertain denervation efficiency by anti-NF staining. Well denervated muscles were then thawed and pooled for immunoblotting. Approximately 40 denervated animals were used for the study. Three tissue-specific molecular forms of BEN protein have been previously identified: a neural form of $95 \mathrm{kDa}$, an intermediate hemopoietic form of $100 \mathrm{kDa}$, and a heavy epithelial form of $110 \mathrm{kDa}$ (Pourquié et al., 1992a). BEN antibodies were tested on Western blots of muscle homogenates and compared with the purified protein extracted from embryonic chick spinal cord and bursa of Fabricius.

Immunoblots show that 4H5 antibody detects a band with an apparent molecular weight of $110 \mathrm{kDa}$ in normal as well as in denervated ALD and PLD muscles (Fig. 4H). Comparison with purified $\mathrm{BEN}$ isoforms indicates that the protein expressed at neuromuscular junctions and in denervated muscle fibers is more similar to the form found in the epithelium of the bursa of Fabricius than to the one expressed in embryonic spinal cord (Fig. $4 H$ ). Immunoblots show that $4 \mathrm{H} 5$ antibody also recognizes a 110 $\mathrm{kDa}$ band in denervated muscles (Fig. $4 H$ ), indicating that the same isoform of BEN protein is present at mature neuromuscular junctions and in muscle fibers after nerve section. The protein is accumulated at a higher level in normal ALD than in PLD, in relation with the particular pattern of innervation of these muscles. As a consequence, the effect of denervation is more significant in the monoinnervated PLD than in the multiinnervated ALD (Fig. 4H).

Our results show that during neuromuscular development, synaptic sites express an isoform of BEN protein that resembles the epithelial form and differs from the neural one. This isoform is more abundant in denervated than in normally innervated muscle fibers.

\section{DISCUSSION}

\section{BEN expression is developmentally regulated during neuromuscular interactions in the chick}

The distribution pattern of BEN shows that at early stages of embryogenesis, it is expressed in both motoneurons and muscle cells. When motor nerves have reached their developing target muscles, the protein disappears from cell bodies of motoneurons and is restricted to nerve terminals. Simultaneously, BEN is progressively downregulated at the muscle membrane to become perfectly colocalized with AChR clusters, so that, during further neuromuscular development, the protein expression only delineates the differentiated synaptic sites

Based on its homophilic properties and its ability to promote neurite outgrowth, BEN was proposed to be involved in the early steps of nerve formation, such as axonal pathfinding and fasciculation. In vitro experiments have shown that sensory, sympathetic, and ciliary neurons, which express BEN, extend neurites on BEN substrate (DeBernardo and Chang, 1995). This mecha- nism may also been involved in fasciculation of peripheral (Pourquié et al., 1990, 1992) and central (Simon et al., 1994) motor axons. The progressive downregulation of BEN protein on both motor neurons and muscle cells and its restrictive localization at synaptic sites, which occurs when nerve-muscle contacts are established, suggest an alternative or additional role for this molecule in the recognition and/or the adhesion between the motor nerve terminal and the target muscle membrane. A role for $\mathrm{BEN}$ as a presumptive target recognition molecule has been already proposed in the chick during the development of the olivocerebellar system (Chedotal et al., 1996) and in the hindbrain where BEN expression has been observed in muscle plate at the time it is contacted by BEN-positive growth cones (Simon et al., 1994).

Supporting this view is the fact that BEN is able to mediate homophilic binding (Tanaka et al., 1991; El-Deeb et al., 1992; DeBernardo and Chang, 1995). Biochemical characterization of the protein has shown that the molecules expressed in neurons, epithelia, and hemopoietic cells differ in their level of $N$-glycosylation, but a recent study demonstrates that it does not affect BEN-binding, homophilic adhesion occurring indifferently between the same or different BEN isoforms (Corbel et al., 1996). We show here that BEN protein accumulated at differentiated neuromuscular junctions differs from the form expressed in embryonic spinal cord and is more similar to the heavy epithelial form purified from the bursal epithelium (Pourquié et al., 1992a; Corbel et al., 1996). Surprisingly, the neural form is not detected at synaptic sites. One explanation for this observation is that BEN expressed in motor nerve terminals is mostly downregulated during development and that a muscular form of the protein persists at differentiated neuromuscular junctions. This interpretation is favored by the fact that BEN monoclonal antibody detects the protein expressed in embryonic motor axons but not the one accumulated in muscle and at mature synaptic sites (Pourquié et al., 1990), which is only detected by 4 H5 antibody. In this case, during the formation of neuromuscular contacts, an interaction would occur between the neural form of BEN expressed by embryonic motor nerve terminals and a form accumulated at the muscle membrane; subsequent differentiation of synaptic sites would be then characterized by a major accumulation of this muscular form at neuromuscular junctions. Alternatively, a shift between two different neural isoforms could take place during development. This has been already described in chick retina in which BEN protein differs between embryo and adult (Pollerberg and Mack, 1994). Consequently, another possibility could be that only an adult neural form of the protein persists in mature motor nerve terminals and that BEN is progressively downregulated at the muscle membrane after the establishment of neuromuscular contacts.

The idea that BEN protein localization at synaptic sites is closely related to the formation of neuromuscular contacts is supported by the fact that BEN is heterogeneously downregu-

\section{$\leftarrow$}

(E, arrowhead). Conversely, BEN protein is reexpressed in regenerating axons (E, arrow). At this time, clustering of AChRs in reinnervated muscle has not yet occurred $(F)$. Simultaneous staining with 4H5 antibody and TRITC- $\alpha$ BGT of a longitudinal section from a reinnervated muscle in a 25 -d-old chicken $(G)$. BEN-positive regenerating nerve terminals progressively contact AChR clusters $(G$, arrows $)$. $H$, Biochemical characterization of BEN by immunoblotting with 4H5 antibody. Lane 1, Normal ALD muscle; lane 2, denervated ALD muscle; lane 3, normal PLD muscle; lane 4, denervated PLD muscle; lane 6, purified protein from bursal epithelia; lane 7, purified protein from embryonic spinal cord. Immunoblots evidence a muscle isoform (lanes 1-4) distinct from the neural purified protein (lane 7) but similar to that expressed in the bursal epithelia (lane 6 ). This isoform is strongly accumulated in denervated muscles (compare lanes 1 and 3 with lanes 2 and 4). Scale bars: $E, F, 125 \mu \mathrm{m} ; A, D, 60 \mu \mathrm{m} ; B, C, 45 \mu \mathrm{m} ; G, 30 \mu \mathrm{m}$. 
lated among fibers in a given muscle. One possible explanation is that it is related to the progressive innervation of muscle cells during myogenesis. The primary wave of myogenesis occurs in brachial muscles from E3 to E6 in ovo while proliferation of secondary myoblasts takes place from E5 to E14 (for review, see McLennan, 1994). Then, from E7, some myotubes in brachial muscles acquire their innervation (Smith and Slater, 1983; Noakes et al., 1986), and BEN would become colocalized at junctional sites, whereas the others are not yet contacted by nerve terminals and would still express BEN on their whole surface.

This interpretation is also favored by the observation that BEN mRNA and protein are not simultaneously downregulated among motoneurons in the ventral horn. A decrease in BEN expression is first observed in motor neurons of the medial motor column (Fig. 3), whose motor axons innervate epaxial muscles $2 \mathrm{~d}$ before the innervation of limb muscles by motor neurons of the lateral motor column (Auda-Boucher et al., 1997), in which a decrease of BEN expression is observed later during development (Fig. 3). This suggests that BEN downregulation, both in motor neurons and muscle cells, is a gradual process that takes place as axons grow and contact their muscle target. A similar dynamic expression of BEN in motor neurons and muscle plate has been already described during development of the chick hindbrain (Simon et al., 1994).

Alternatively, one could argue that BEN is restricted to subsets of embryonic motor neurons and/or muscle fibers, like in the developing olivocerebellar system, where BEN-expressing climbing fibers synapse on BEN-negative Purkinje cells (Chedotal et al., 1996). In this case, heterophilic interactions must occur between BEN and other still unknown receptor molecules. It has been recently shown that $\mathrm{BEN}$ is able to bind CD6, a member of the cysteine-rich scavenger receptor family (Bowen et al., 1995, 1997) and $\mathrm{Ng}-\mathrm{CAM}$, another member of the Ig superfamily (DeBernardo and Chang, 1996). However, at least for muscle cells, our in vitro results do not favor a restrictive pattern of expression to some of them. Indeed, myoblasts cultivated from dorsal and ventral muscle masses from the forelimb all express BEN, whatever their origin. In addition, all muscle cells grown in vitro maintain BEN expression even when they fuse and give rise to mature myotubes. Consequently, we think that BEN heterogeneous downregulation during embryonic myogenesis is closely related to the progressive innervation of muscle cells.

\section{BEN expression in muscle is nerve-regulated during embryonic and postnatal development of the chick}

Our in vitro data, together with the neural tube ablation and denervation experiments, show that all myogenic cells intrinsically express BEN and maintain the protein along the muscle membrane in the absence of innervation. Conversely, when neurons are added to myogenic cultures or denervated muscles allowed to be reinnervated, BEN protein is expressed in growing motor axons and rapidly downregulated in myotubes, demonstrating that innervation controls the restrictive pattern of BEN expression during the establishment of neuromuscular connections.

Such a nerve-dependent regulation of cell adhesion molecules in muscle has been already described for $\mathrm{N}$-CAM and $\mathrm{N}$-cadherin, which are both expressed in developing muscles at the time of nerve trunk ingrowth, downregulated after synaptogenesis, and reexpressed at the surface of denervated muscles (Rieger et al., 1985; Covault and Sanes, 1986; Cifuentes-Diaz et al., 1994).
Other cell surface molecules are also observed in close association with the synaptic basal lamina when it develops, as $S$-laminin (Hunter et al., 1989) and heparan sulfate proteoglycan (Peng et al., 1995), which are proposed to be involved in retrograde signaling from the muscle to the nerve after their initial contact (for review, see Ruegg and Bixby, 1998). Although the precise role of $\mathrm{BEN}$ protein in the molecular events leading to the formation of neuromuscular synapses remains to be determined, our coculture and denervation/reinnervation experiments show that BEN protein restriction at synaptic sites precedes the appearance of AChR clusters, which in both cases (cocultures and reinnervated muscle) takes place after the downregulation of BEN in muscle. However, it seems unlikely that BEN restriction at the neuromuscular junction could be one of the events governing AChR clustering in the postsynaptic membrane. Indeed, in developing chick embryo, AChR clusters are induced to form on myotubes around intramuscular nerve trunks without any direct contact with nerve profiles (Dahm and Landmesser, 1991; Auda-Boucher et al., 1997). In addition, spontaneous clustering of AChR occurs on chick myotubes grown in vitro (Fischbach and Cohen, 1973; Anderson and Cohen, 1977; Lefeuvre et al., 1996), and colocalization of BEN protein with AChR clusters was never observed in our muscle cell cultures grown without neurons (data not shown).

The dynamic regulation of CAM expression during neurontarget interaction has led to some investigations concerning the activity-dependent modulation of these molecules. In chick, a role for electrical activity in regulating neuronal PSA-NCAM expression was shown by in ovo injection of curare, resulting in correlated increases in PSA-NCAM, intramuscular nerve branching, and synaptogenesis (Landmesser et al., 1990; Bruses et al., 1995). Studies on cultured myotubes have also shown that PSA-NCAM synthesis in muscle is closely coupled to the level of electrical activity, being enhanced by stimulation or altered by treatment with tetrodotoxin (Rafuse and Landmesser, 1996).

Previous studies in chick (Oppenheim et al., 1989; Dahm and Landmesser, 1991; Fournier Le Ray et al., 1993) have shown that inactivity produced by neuromuscular blockade induces axonal branching and synaptogenesis, which, in turn, promotes motoneuron survival. Conversely, an increased motoneuron activity provoked by chronic spinal cord stimulation does not modify motoneuron number but induces, after the cell death period, a terminal sprouting of nerve terminals, resulting in an increase in the number of neuromuscular synapses (Fournier Le Ray and Fontaine-Perus, 1991). However, both inhibition and stimulation of neuromuscular activity do not change the pattern of BEN protein expression, BEN appearing colocalized in both cases at supernumerary synapses (our observations; see Fournier Le Ray and Fontaine-Perus, 1991). In addition, although the effect of tetrodotoxin was not studied in our in vitro experiments, spontaneous contractions were observed in chick myotubes from day 5 of culture (see also Rafuse and Landmesser, 1996), and no modification in the distribution of BEN protein was observed in these conditions (see Results). Because activity-dependent changes during the establishment of neuromuscular innervation mainly concerns intramuscular nerve branching, it could be argued that $\mathrm{BEN}$ is downstream of these mechanisms and consequently not directly regulated by electrical activity.

In conclusion, the precise spatiotemporal expression of BEN during neuromuscular development and the experimental manipulations reported here support the contention that BEN could 
participate in the events leading to the recognition between nerve and muscle both in the formation of synaptic contacts and during muscle reinnervation.

\section{REFERENCES}

Anderson MJ, Cohen MW (1977) Nerve-induced and spontaneous redistribution of acetylcholine receptors on cultured muscle cells. J Physiol (Lond) 268:757-773.

Auda-Boucher G, Jarno V, Fournier-Thibault C, Butler-Browne G, Fontaine-Perus J (1997) Acetylcholine receptor formation in mouse chick chimera. Exp Cell Res 236:29-42.

Bowen MA, Patel DD, Li X, Modrell AR, Malacko AR, Wang WC, Marquardt H, Neubauer M, Pesando JM, Francke U (1995) Cloning, mapping and characterization of activated leucocyte-cell adhesion molecule (ALCAM), a CD6 ligand. J Exp Med 181:2213-2220.

Bowen MA, Bajorath J, D'Egidio M, Whitney GS, Palmer D, Kobarg J, Starling GC, Siadak AW, Aruffo A (1997) Characterization of mouse ALCAM(CD166): the CD6-binding domain is conserved in different homologs and mediates cross-species binding. Eur $\mathrm{J}$ Immunol 27:1469-1478.

Bruses JL, Oka S, Rutishauser U (1995) NCAM-associated polysialic acid on ciliary ganglion neurons is regulated by polysialyltransferase levels and interaction with muscle. J Neurosci 15:8310-8319.

Burns FR, Von Kannen S, Guy L, Raper JA, Kamholz J, Chang S (1991) DM-GRASP, a novel immunoglobulin superfamily axonal surface protein that supports neurite extension. Neuron 7:209-220.

Chedotal A, Pourquié O, Ezan F, San Clemente H, Sotelo C (1996) $\mathrm{BEN}$ as a presumptive target recognition molecule during the development of the olivocerebellar system. J Neurosci 16:3296-3310.

Chiba A, Keshishian H (1996) Neuronal pathfinding and recognition: roles of cell adhesion molecules. Dev Biol 180:424-432.

Cifuentes-Diaz C, Nicolet M, Goudou D, Rieger F, Mege RM (1994) $N$-Cadherin expression in developing, adult and denervated chicken neuromuscular system: accumulation at both the neuromuscular junction and the node of Ranvier. Development 120:1-11.

Corbel C, Bluestein H, Pourquié O, Vaigot P, Le Douarin, NM (1992a) An antigen expressed by avian neuronal cells is also expressed by activated T lymphocytes. Cell Immunol 141:99-110.

Corbel C, Cormier F, Pourquié O, Bluestein H (1992b) BEN, a novel surface molecule of the immunoglobulin superfamily on the avian hemopoietic progenitor cells shared with neural cells. Exp Cell Res 203:91-99.

Corbel C, Pourquié O, Cormier F, Vaigot P, Le Douarin NM (1996) BEN/SC1/DM-GRASP, a homophilic adhesion molecule, is required for in vitro myeloid formation by avian hemopoietic progenitors. Proc Natl Acad Sci USA 93:2844-2849.

Covault J, Sanes J (1986) Distribution of NCAM in synaptic and extrasynaptic portions of developing and adult skeletal muscle. J Cell Biol 102:731-739.

Dahm L, Landmesser L (1991) The regulation of synaptogenesis during normal development and following activity blockade. J Neurosci $11: 238-255$.

DeBernardo AP, Chang S (1995) Native and recombinant DM-GRASP selectively support neurite extension from neurons that express GRASP. Dev Biol 169:65-75.

DeBernardo AP, Chang S (1996) Heterophilic interactions of DMGRASP: grasp-NgCam interactions involved in neurite extension. J Cell Biol 133:657-666.

Eichmann A, Marcelle C, Breant C, Le Douarin NM (1993) Two novel molecules related to the VGEF-receptor are expressed in early endothelial cells during avian embryonic development. Mech Dev 42:33-48.

El-Deeb S, Thompson SC, Covault J (1992) Characterization of a cell surface adhesion molecule expressed by a subset of developing chick neurons. Dev Biol 149:213-227.

Fallon JR, Gelfman CE (1989) Agrin-related molecules are concentrated at acetylcholine receptor clusters in normal and aneural developing muscle. J Cell Biol 108:1527-1535.

Fields RD, Itoh K (1996) Neural cell adhesion molecules in activitydependent development and synaptic plasticity. Trends Neurosci 19:473-480.

Fischbach GD, Cohen SA (1973) The distribution of acetylcholine sensitivity over uninnervated and innervated muscle fibres grown in cell culture. Dev Biol 31:147-162.

Fournier Le Ray C, Fontaine-Perus J (1991) Influence of spinal cord stimulation on the innervation pattern of muscle fibers in vivo. J Neurosci 11:3840-3850.

Fournier Le Ray C, Prevette D, Oppenheim RW, Fontaine-Perus J (1993) Interactions between spinal cord stimulation and activity blockade in the regulation of synaptogenesis and motoneuron survival in the chick embryo. J Neurobiol 24:1142-1156.

Fredette BJ, Ranscht B (1994) T-Cadherin expression delineates specific regions of the developing motor axon-hindlimb projection pathway. J Neurosci 14:7331-7346.

Ginsborg BL, Mackay B (1960) The Latissimus dorsi of the chick. J Physiol (Lond) 153:19-20.

Hall ZW, Sanes JR (1993) Synaptic structure and development: the neuromuscular junction. Cell [Suppl] 72:99-121.

Hamburger V, Hamilton H (1951) A series of normal stages in the development of the chick embryo. J Morphol 99:49-92.

Henrique D, Adam J, Myat A, Chitnis A, Lewis J, Hish-Horowicz D (1995) Expression of a delta homologue in prospective neurons of the chick. Nature 375:787-790.

Hunter DD, Shah V, Merlie JP, Sanes JR (1989) A laminin like adhesive protein concentrated in the synaptic cleft of the neuromuscular junction. Nature 338:229-234.

Keynes R, Stern C (1984) Segmentation in the vertebrate nervous system. Nature 310:786-789.

Keynes R, Stern C (1988) Mechanisms of vertebrate segmentation. Development 103:413-429.

Khaskiye A (1986) Effects of denervation and direct electrical stimulation upon acetylcholine receptors and acetylcholinesterase accumulation in developing latissimus dorsi muscle of the chick. Biol. Cell $58: 245-250$

Landmesser L, Dahm L, Tang J, Rutishauser U (1990) Polysialic acid as a regulator of intramuscular nerve branching during embryonic development. Neuron 4:655-667.

Lefeuvre B, Crossin F, Fontaine-Perus J, Bandmann E, Gardahaut MF (1996) Innervation regulates myosin heavy chain isoform expression in developing skeletal muscle fibers. Mech Dev 58:115-127.

Martini R, Schachner M (1991) Complex expression pattern of tenascin during innervation of the posterior limb buds of the developing chicken. J Neurosci Res 28:261-279.

McLennan IS (1994) Neurogenic and myogenic regulation of skeletal muscle formation: a critical re-evaluation. Prog Neurobiol 44:119-140.

Noakes PG, Everett AW, Bennett MR (1986) The growth of muscle nerves in relation to the formation of primary myotubes in the developing chick forelimb. J Comp Neurol 248:245-256.

Oppenheim RW, Bursztajn S, Prevette D (1989) Cell death of motoneurons in the chick embryo spinal cord: acetylcholine receptors and synaptogenesis in skeletal muscle following the reduction of motoneuron cell death by neuromuscular blockade. Development 107:331-341.

Peng HB, Ali AA, Dai Z, Daggett DF, Raulo E, Rauvala H (1995) The role of heparin-binding growth-associated molecule (HB-GAM) in the postsynaptic induction in cultured muscle cells. $\mathrm{J}$ Neurosci 15:3027-3038.

Pollerberg EG, Mack TGA (1994) Cell adhesion molecule SC1/DMGRASP is expressed on growing axons of retina ganglion cells and is involved in mediating their extension on axons. Dev Biol 165:670-687.

Pourquié O, Coltey M, Thomas J, Le Douarin NM (1990) A widely distributed antigen developmentally regulated in the nervous system. Development 109:743-752.

Pourquié O, Corbel C, Le Caer JP, Rossier J, Le Douarin NM (1992a) $\mathrm{BEN}$, a surface molecule of the immunoglobulin superfamily expressed in a variety of developing systems. Proc Natl Acad Sci USA 89:5261-5265.

Pourquié O, Hallonet MER, Le Douarin NM (1992b) Ben glycoprotein expression is associated to climbing fibers axonogenesis in the avian cerebellum. J Neurosci 12:1548-1557.

Rafuse VF, Landmesser L (1996) Contractile activity regulates isoform expression and polysialilation of NCAM in cultured myotubes: involvement of $\mathrm{Ca}^{2+}$ and protein kinase C. J Cell Biol 132:969-983.

Rieger F, Grumet M, Edelman GM (1985) N-CAM at the vertebrate neuromuscular junction. J Cell Biol 101:285-293.

Rong PM, Teillet MA, Ziller C, Le Douarin NM (1992) The neural tube/notochord complex is necessary for vertebral but not limb and body wall striated muscle differentiation. Development 115:657-672.

Ruegg MA, Bixby JL (1998) Agrin orchestrates synaptic differentiation at the vertebrate neuromuscular junction. Trends Neurosci 21:22-27.

Schuster CM, Davis GW, Fetter RD, Goodman CS (1996) Genetic 
dissection of structural and functional components of synaptic plasticity. I. Fasciclin II controls synaptic stabilization and growth. Neuron 17:641-654.

Simon H, Guthrie S, Lumsden A (1994) Regulation of SC1/DMGRASP during the migration of motor neurons in the chick embryo brain stem. J Neurobiol 25:1129-1143.

Smith MA, Slater CR (1983) Spatial distribution of acetylcholine receptors at developing chick neuromuscular junctions. J Neurocytol 12:993-1005.

Strähle U, Blader P, Adam J, Ingham PN (1994) A simple and efficience procedure for non-isotopic in situ hybridization to sectioned material. Trends Genet 10:75-76.

Tanaka H, Obata K (1984) Developmental changes in unique cell sur- face antigens of chick embryo spinal motorneurons and ganglion cells. Dev Biol 106:26-37.

Tanaka H, Matsui T, Agata A, Tomura M, Kubota J, McFarland KC, Kohr B, Lee A, Phillips HS, Shelton, DL (1991) Molecular cloning and expression of a novel adhesion molecule, SC1. Neuron 7:535-545.

Tang J, Rutishauser U, Landmesser L (1994) Polysialic acid regulates growth cone behavior during sorting of motor axons in the plexus region. Neuron 13:405-414.

Tosney KW (1987) Growth cone interactions with anterior and posterior sclerotome. Soc Neurosci Abstr 13:253.

Tosney KW (1988) Proximal tissues and patterned neurite outgrowth at the lumbosacral level of the chick embryo: partial and complete deletion of the somite. Dev Biol 127:266-286. 